\title{
General boundary value problems for pseudo-differential equations and related difference equations
}

Vladimir B Vasilyev ${ }^{*}$

${ }^{*}$ Correspondence:

vladimir.b.vasilyev@gmail.com Chair of Pure Mathematics, Lipetsk State Technical University, Moskovskaya 30, Lipetsk, 398600 Russia

\begin{abstract}
The author develops the theory of pseudo-differential equations and boundary value problems in non-smooth domains. A model pseudo-differential equation in a canonical flat domain is reduced to a system of linear difference equations.

MSC: $35 \mathrm{~S} 15 ; 39 \mathrm{~A} 05$

Keywords: general boundary value problem; linear integral equation; system of difference equations
\end{abstract}

\section{Introduction}

It is well known that the term 'elliptic boundary value problem' means not only satisfying certain equation in inner points of a manifold, but satisfying some boundary conditions as well. But it is not enough. These boundary conditions have been assiciated with inner equation, and these conditions of concordance are called Shapiro-Lopatinskii conditions. Indeed, this condition is a non-vanishing determinant of a certain system of linear algebraic equations. But it is true for smooth boundary only, because it may be locally straightened by diffeomorphism transforming to a hyper-plane. So, locally, one considers a model problem with frozen coefficients in a half-space. Originating boundary conditions are determined by an infinite number of solutions, which are linear combinations of some arbitrary functions. For unique defining of these functions, one needs certain additional conditions. Such conditions are more usable as traces of certain pseudo-differential operators on a boundary. Of course, there is a possibility for other kinds of boundary conditions. This phenomenon was explained mathematically in papers by Vishik and Eskin (see [1]) using the concept of factorization for an elliptic symbol of a pseudo-differential operator. The author tried to apply this idea to a multidimensional situation and non-smooth boundary [2], and for some cases, typical analogues of the mentioned results were obtained. However, for general boundary conditions, this problem is very hard, and maximum that we can obtain is the equivalent system of linear difference equations for defining arbitrary functions. This is the main conclusion of this paper.

One can find many other approaches to the theory of boundary value problems on manifolds with non-smooth boundary in [3-5].

O2013 Vasilyev; licensee Springer. This is an Open Access article distributed under the terms of the Creative Commons Attribution License (http://creativecommons.org/licenses/by/2.0), which permits unrestricted use, distribution, and reproduction in any medium, provided the original work is properly cited. 


\section{General boundary value problem}

Let $A$ be a pseudo-differential operator with symbol $A(\xi), \xi=\left(\xi_{1}, \xi_{2}\right)$ which satisfies the ellipticity condition:

$$
c_{1} \leq\left|A(\xi)(1+|\xi|)^{-\alpha}\right| \leq c_{2}, \quad \alpha \in \mathbf{R},
$$

where $c_{1}, c_{2}$ are positive constants.

By wave factorization of $A(\xi)$ with respect to the cone $C_{+}^{a}=\left\{x=\left(x_{1}, x_{2}\right) \in \mathbf{R}^{2}: x_{2}>\right.$ $\left.a\left|x_{1}\right|, a>0\right\}$, we mean its representation in the form

$$
A(\xi)=A_{\neq}(\xi) A_{=}(\xi)
$$

where the factors $A_{\neq}(\xi), A_{=}(\xi)$ must satisfy the following conditions:

(1) $A_{\neq}(\xi)$ is defined, generally speaking, on the set $\left\{x \in \mathbf{R}^{2}: a^{2} x_{2}^{2} \neq x_{1}^{2}\right\}$ only;

(2) $A_{\neq}(\xi)$ admits an analytical continuation into the radial tube domain $T\left(C_{+}^{*}\right)$ over the cone $\stackrel{*}{C}_{+}^{a}=\left\{x \in \mathbf{R}^{2}: a x_{2}>\left|x_{1}\right|\right\}$, which satisfies the following estimate:

$$
\left|A_{\neq}^{ \pm 1}(\xi+i \tau)\right| \leq c(1+|\xi|+|\tau|)^{ \pm \mathfrak{x}}, \quad \forall \tau \in C_{+}^{*} .
$$

Similar properties must have the factor $A_{=}(\xi)$ with change $-\stackrel{*}{C_{+}^{a}}$ instead of $\stackrel{*}{C}_{+}^{a}$ and $\alpha-\mathfrak{x}$ instead of æ.

The number $æ$ is called an index of wave factorization of $A(\xi)$ with respect to the cone $C_{+}^{a}$.

A convenient scale of spaces for studying pseudo-differential equations in infinite angle $C_{+}^{a}$ is the scale of Sobolev-Slobodetskii spaces. By definition, the space $H^{s}\left(\mathbf{R}^{2}\right), s \in \mathbf{R}$, consists of (generalized) functions $u(x)$ for which their Fourier transforms $u(\xi)$ are local integrable in Lebesgue sense functions, and the norm is finite:

$$
\|u\|_{s}^{2}=\int_{\mathbf{R}^{2}}|\tilde{u}(\xi)|^{2}(1+|\xi|)^{2 s} d \xi<+\infty
$$

The space $H^{s}\left(C_{+}^{a}\right)$ consists of functions from $H^{s}\left(\mathbf{R}^{2}\right)$ with support in $\bar{C}_{+}^{a}$ (closure of $\left.C_{+}^{a}\right)$. We are interested in solving the equation

$$
(A u)(x)=f(x), \quad x \in C_{+}^{a},
$$

in the space $H^{s}\left(C_{+}^{a}\right)$ if the right-hand side is taken from the space $H_{1}^{s-\alpha}\left(C_{+}^{a}\right)$ of $S^{\prime}\left(C_{+}^{a}\right)$ distributions which admit a continuation $l u$ on the whole $\mathbf{R}^{2}$, and this continuation belongs to $H^{s-\alpha}\left(\mathbf{R}^{2}\right)$. The norm in $H_{1}^{s-\alpha}\left(C_{+}^{a}\right)$ is defined by formula $\|u\|_{H_{1}^{s-\alpha}\left(C_{+}^{a}\right)} \equiv\|u\|_{s-\alpha}^{+}=\inf \|l u\|_{s-\alpha}$. We suppose the symbol $A(\xi)$ admits the wave factorization.

For functions from Schwartz class (infinitely differentiable rapidly decreasing at infinity), we define the integral operator

$$
(G u)=\lim _{\tau \rightarrow 0} \int_{\mathbf{R}^{2}} \frac{u\left(y_{1}, y_{2}\right) d y}{\left(x_{!}-y_{1}\right)^{2}-a^{2}\left(a^{2}-y_{2}+i \tau\right)^{2}}, \quad x \in \mathbf{R}^{2} .
$$

The following theorem is valid [2]. 
Theorem 1 Let $\mathfrak{x}-s=n+\delta, n>0, n \in \mathbf{Z},|\delta|<1 / 2$. Then equation (1) for an arbitrary right-hand side $f \in H_{1}^{s-\alpha}\left(C_{+}^{a}\right)$ has solutions from $H^{s}\left(C_{+}^{a}\right)$, and their Fourier transforms are written by formula

$$
\begin{aligned}
\tilde{u}(\xi)= & A_{\neq}^{-1}(\xi) \times\left(Q(\xi) G Q^{-1} A_{\neq}^{-1} \tilde{l} f\right. \\
& \left.+\sum_{k=0}^{n-1}\left(\tilde{c}_{k}\left(\xi_{1}-a \xi_{2}\right)\left(\xi_{1}+a \xi_{2}\right)^{k}+\tilde{d}_{k}\left(\xi_{1}+a \xi_{2}\right)\left(\xi_{1}-a \xi_{2}\right)^{k}\right)\right),
\end{aligned}
$$

where $c_{k}, d_{k}$ are arbitrary functions from $H^{s_{k}}\left(\mathbf{R}_{-}\right), H^{s_{k}}\left(\mathbf{R}_{+}\right)$, respectively, $\mathbf{R}_{+}=\{x \in \mathbf{R}$ : $\pm x>0\}, s_{k}=s-\mathfrak{x}+k+1 / 2, k=0,1, \ldots, n-1, Q$ is arbitrary polynomial satisfying the estimate ( $*$ ) for $\alpha=n$, and formula (2) describes all possible solutions of equation (1).

The solution (2) depends on $2 n$ arbitrary functions $c_{k}, d_{k}$, and to remove this nonuniqueness of solution, we will give $n$ boundary conditions on every angle sides $\partial C_{+}^{a}$ in the following form:

$$
\left.\left(B_{1 j} u\right)\right|_{\substack{x_{1}>0 \\ x_{2}-a x_{1}=0}}=g_{1 j} ;\left.\quad\left(B_{2 j} u\right)\right|_{\substack{1_{1}<0 \\ x_{2}-a x_{1}=0}}=g_{2 j}
$$

where $B_{r j}, r=1,2$, are pseudo-differential operators with symbols $B_{r j}(\xi)$ of homogeneity order $\beta_{r, j}$ satisfying the estimates

$$
\left|B_{r j}(\xi)\right| \leq c|\xi|^{\beta_{r j}} ; \quad 0 \leq \beta_{r j} \leq s-1 / 2 .
$$

Passing to Fourier images in (3), previously making the linear change of variables, which transfers $C_{+}^{a}$ to the first quadrant, we have

$$
\begin{aligned}
& \int_{-\infty}^{+\infty} B_{1 j} \tilde{U}(\xi) d \xi_{2}=\tilde{g}_{1 j}\left(\xi_{1}\right) ; \quad \int_{-\infty}^{+\infty} B_{2 j} \tilde{U}(\xi) d \xi_{1}=\tilde{g}_{2 j}\left(\xi_{2}\right) ; \\
& B_{r j}^{\prime}(\xi)=B_{r j}\left(\frac{\xi_{1}-\xi_{2}}{2 a}, \frac{\xi_{1}+\xi_{2}}{a}\right), \quad \tilde{U}(\xi)=\tilde{u}\left(\frac{\xi_{1}-\xi_{2}}{2 a}, \frac{\xi_{1}+\xi_{2}}{a}\right) .
\end{aligned}
$$

Then, with the help of the same change, we will write the general solution of equation (2) in the form

$$
\begin{aligned}
& \tilde{U}(\xi)=F(\xi)+a_{\neq}^{-1}(\xi) \sum_{k=0}^{n-1}\left(\tilde{c}_{k}\left(\xi_{1}\right) \xi_{2}^{k}+\tilde{d}_{k}\left(\xi_{2}\right) \xi_{1}^{k}\right), \\
& F(\xi)=\left(A_{\neq}^{-1} Q G Q^{-1} A_{\neq}^{-1} \tilde{l} f\right)\left(\frac{\xi_{1}-\xi_{2}}{2 a}, \frac{\xi_{1}+\xi_{2}}{a}\right), \\
& a_{\neq}^{-1}(\xi)=A_{\neq}^{-1}\left(\frac{\xi_{1}-\xi_{2}}{2 a}, \frac{\xi_{1}+\xi_{2}}{a}\right) .
\end{aligned}
$$

Substituting $\tilde{U}(\xi)$ into equations (4), we obtain the following system:

$$
\left\{\begin{array}{l}
\sum_{n=0}^{n-1} b_{1 j k}\left(\xi_{1}\right)\left[\tilde{c}_{k}\left(\xi_{1}\right)+\int_{-\infty}^{+\infty} K_{1 j k}\left(\xi_{1}, \xi_{2}\right) \tilde{d}_{k}\left(\xi_{2}\right) d \xi_{2}\right]=\tilde{g}_{1 j}^{\prime}\left(\xi_{1}\right)-F_{1 j}\left(\xi_{1}\right), \\
\sum_{n=0}^{n-1} b_{2 j k}\left(\xi_{2}\right)\left[\tilde{d}_{k}\left(\xi_{2}\right)+\int_{-\infty}^{+\infty} K_{2 j k}\left(\xi_{1}, \xi_{2}\right) \tilde{c}_{k}\left(\xi_{1}\right) d \xi_{1}\right]=\tilde{g}_{2 j}^{\prime}\left(\xi_{1}\right)-F_{2 j}\left(\xi_{1}\right),
\end{array}\right.
$$


where

$$
\begin{aligned}
& F_{1 j}\left(\xi_{1}\right)=\int_{-\infty}^{+\infty} B_{1 j}^{\prime}(\xi) F(\xi) d \xi_{2} ; \quad F_{2 j}\left(\xi_{2}\right)=\int_{-\infty}^{+\infty} B_{2 j}^{\prime}(\xi) F(\xi) d \xi_{1}, \\
& b_{1 j k}\left(\xi_{1}\right)=\int_{-\infty}^{+\infty} B_{1 j}^{\prime}(\xi) a_{\neq}^{-1}(\xi) \xi_{2}^{k} d \xi_{2} ; \quad b_{2 j k}\left(\xi_{2}\right)=\int_{-\infty}^{+\infty} B_{2 j}^{\prime}(\xi) a_{\neq}^{-1}(\xi) \xi_{1}^{k} d \xi_{1}, \\
& K_{r j k}(\xi)=\xi_{r}^{k} b_{r j k}^{-1}\left(\xi_{r}\right) B_{r j}^{\prime}(\xi) a_{\neq}^{-1}(\xi), \quad r=1,2 .
\end{aligned}
$$

Let us note that the kernels $K_{r j k}(\xi)$ in the system of integral equations (5) are homogeneous of order -1 . Indeed,

$$
\begin{gathered}
b_{1 j k}\left(l \xi_{1}\right) \int_{-\infty}^{+\infty} B_{1 j}^{\prime}\left(l \xi_{1}, \xi_{2}\right) a_{\neq}^{-1}\left(l \xi_{1}, \xi_{2}\right) \xi_{2}^{k} d \xi_{2}=\int_{-\infty}^{+\infty} B_{1 j}^{\prime}\left(l \xi_{1}, l \xi_{2}\right) a_{\neq}^{-1}\left(l \xi_{1}, l \xi_{2}\right)\left(l \xi_{2}\right)^{k} l d \xi_{2} \\
=l^{\beta_{1} j} l^{-\mathfrak{x}} l^{k} l b_{1 j k}\left(\xi_{1}\right)=l^{b_{1 j}-\mathfrak{x}+k+1} b_{1 j k}\left(\xi_{1}\right) ; \\
K_{1 j k}\left(l \xi_{1}, l \xi_{2}\right)=\left(l \xi_{1}\right)^{k} l^{-b_{1}+\mathfrak{x}+k+1} b_{1 j k}^{-1}\left(\xi_{1}\right) B_{1 j}(l \xi) a_{\neq}^{-1}(l \xi) \\
=l^{-\beta_{1 j}+\mathfrak{x}-k-1} l^{\beta_{1 j}} l^{-\mathfrak{x}} l^{k} \xi_{1}^{k} b_{1 j k}^{-1}(\xi) B_{1 j}^{\prime}(\xi) a_{\neq}^{-1}(\xi)=l^{-1} K_{1 j k}(\xi)
\end{gathered}
$$

and, analogously, for $K_{2 j k}$.

\section{Originating difference equations}

So, we have obtained the system of $2 n$ linear integral equations with $2 n$ unknowns; moreover, kernels of these equations are homogeneous of order -1 . To apply the Mellin transform [6], we introduce the following notations. For all $\xi_{1}>0, \xi_{2}>0$, let

$$
\begin{aligned}
& K_{r j k}^{(11)}\left(\xi_{1}, \xi_{2}\right)=K_{r j k}\left(\xi_{1}, \xi_{2}\right), \quad K_{r j k}^{(12)}\left(\xi_{1}, \xi_{2}\right)=K_{r j k}\left(-\xi_{1}, \xi_{2}\right), \\
& K_{r j k}^{(21)}\left(\xi_{1}, \xi_{2}\right)=K_{r j k}\left(-\xi_{1},-\xi_{2}\right), \quad K_{r j k}^{(22)}\left(\xi_{1}, \xi_{2}\right)=K_{r j k}\left(\xi_{1},-\xi_{2}\right),
\end{aligned}
$$

and then $c_{k}^{(0)}(x)$ denotes the restriction $\tilde{c}_{k}(x), x \in \mathbf{R}$, on $(0 ;+\infty) ; c_{k}^{(1)}(x)$ is the restriction $\tilde{c}_{k}(-x)(0 ;+\infty)$. Analogously, we define $d_{k}^{(0)}(x), d_{k}^{(1)}(x), g_{r j}^{(0)}\left(\xi_{r}\right), g_{r j}^{(1)}\left(\xi_{r}\right), F_{r j}^{(0)}\left(\xi_{r}\right), F_{r j}^{(1)}\left(\xi_{r}\right)$.

Let us consider the equations from system (5) on a half-line: $\left(\xi_{2}>0, \xi_{2}<0\right)$ for the first equation, and $\left(\xi_{1}>0, \xi_{1}<0\right)$ for the second one. In the equations of system (5), we have the following integrals:

$$
\int_{-\infty}^{+\infty} K_{1 j k}\left(\xi_{1}, \xi_{2}\right) \tilde{d}_{k}\left(\xi_{2}\right) d \xi_{2}, \quad \int_{-\infty}^{+\infty} K_{2 j k}\left(\xi_{1}, \xi_{2}\right) \tilde{c}_{k}\left(\xi_{1}\right) d \xi_{1}
$$

for which it is necessary to represent them as a sum of integrals on a half-line.

$$
\begin{aligned}
& \int_{-\infty}^{+\infty} K_{1 j k}\left(\xi_{1}, \xi_{2}\right) \tilde{d}_{k}\left(\xi_{2}\right) d \xi_{2} \\
& \quad=\int_{0}^{+\infty} K_{1 j k}\left(\xi_{1}, \xi_{2}\right) \tilde{d}_{k}\left(\xi_{2}\right) d \xi_{2}+\int_{-\infty}^{0} K_{1 j k}\left(\xi_{1}, \xi_{2}\right) \tilde{d}_{k}\left(\xi_{2}\right) d \xi_{2} \\
& \quad=\int_{0}^{+\infty} K_{1 j k}\left(\xi_{1}, \xi_{2}\right) \tilde{d}_{k}\left(\xi_{2}\right) d \xi_{2}-\int_{-\infty}^{0} K_{1 j k}\left(\xi_{1},-\xi_{2}\right) \tilde{d}_{k}\left(-\xi_{2}\right) d \xi_{2} \\
& \quad=\int_{0}^{+\infty} K_{1 j k}\left(\xi_{1}, \xi_{2}\right) \tilde{d}_{k}\left(\xi_{2}\right) d \xi_{2}+\int_{0}^{+\infty} K_{1 j k}\left(\xi_{1},-\xi_{2}\right) \tilde{d}_{k}\left(-\xi_{2}\right) d \xi_{2} .
\end{aligned}
$$


One can also use analogous arguments for the second integral.

Taking into account these facts, we transform system (5) to a system of $4 n$ linear nonhomogeneous integral equations on a half-axis of type

$$
\left\{\begin{aligned}
g_{1 j}^{(0)}\left(\xi_{1}\right)-F_{1 j}^{(0)}\left(\xi_{1}\right)= & \sum_{k=0}^{n-1} b_{1 j k}\left(\xi_{1}\right)\left[c_{k}^{(0)}\left(\xi_{1}\right)+\int_{0}^{+\infty} K_{1 j k}^{(11)}\left(\xi_{1}, \xi_{2}\right) d_{k}^{(0)}\left(\xi_{2}\right) d \xi_{2}\right. \\
& \left.+\int_{0}^{+\infty} K_{1 j k}^{(22)}\left(\xi_{1}, \xi_{2}\right) d_{k}^{(1)}\left(\xi_{2}\right) d \xi_{2}\right] \\
g_{1 j}^{(1)}\left(\xi_{1}\right)-F_{1 j}^{(1)}\left(\xi_{1}\right)= & \sum_{k=0}^{n-1} b_{1 j k}\left(-\xi_{1}\right)\left[c_{k}^{(1)}\left(\xi_{1}\right)+\int_{0}^{+\infty} K_{1 j k}^{(12)}\left(\xi_{1}, \xi_{2}\right) d_{k}^{(0)}\left(\xi_{2}\right) d \xi_{2}\right. \\
& \left.+\int_{0}^{+\infty} K_{1 j k}^{(21)}\left(\xi_{1}, \xi_{2}\right) d_{k}^{(1)}\left(\xi_{2}\right) d \xi_{2}\right] \\
g_{2 j}^{(0)}\left(\xi_{2}\right)-F_{2 j}^{(0)}\left(\xi_{2}\right)= & \sum_{k=0}^{n-1} b_{2 j k}\left(\xi_{2}\right)\left[d_{k}^{(0)}\left(\xi_{2}\right)+\int_{0}^{+\infty} K_{2 j k}^{(11)}\left(\xi_{1}, \xi_{2}\right) c_{k}^{(0)}\left(\xi_{1}\right) d \xi_{1}\right. \\
& \left.+\int_{0}^{+\infty} K_{2 j k}^{(12)}\left(\xi_{1}, \xi_{2}\right) c_{k}^{(1)}\left(\xi_{1}\right) d \xi_{1}\right] \\
g_{2 j}^{(1)}\left(\xi_{2}\right)-F_{2 j}^{(1)}\left(\xi_{2}\right)= & \sum_{k=0}^{n-1} b_{2 j k}\left(-\xi_{2}\right)\left[d_{k}^{(1)}\left(\xi_{2}\right)+\int_{o}^{+\infty} K_{2 j k}^{(22)}\left(\xi_{1}, \xi_{2}\right) c_{k}^{(0)}\left(\xi_{1}\right) d \xi_{1}\right. \\
& \left.+\int_{0}^{+\infty} K_{2 j k}^{(21)}\left(\xi_{1}, \xi_{2}\right) c_{k}^{(1)}\left(\xi_{1}\right) d \xi_{1}\right] .
\end{aligned}\right.
$$

Let us note that the functions $b_{r j k}\left(\xi_{r}\right), r=1,2$, are homogeneous of order $\gamma_{j k}, \delta_{j k}$ and have the following form:

$$
b_{1 j k}\left(\xi_{1}\right)=\left\{\begin{array}{ll}
A_{j k} \xi_{1}^{\gamma_{j k}}, & \xi_{1}>0, \\
B_{j k}\left(-\xi_{1}\right)^{\gamma_{j k},} & \xi_{1}<0 ;
\end{array} \quad b_{2 j k}\left(\xi_{2}\right)= \begin{cases}C_{j k} \xi_{2}^{\delta_{j k}}, & \xi_{2}>0, \\
D_{j k}\left(-\xi_{2}\right)^{\delta_{j k},} & \xi_{2}<0,\end{cases}\right.
$$

where $\gamma_{j k}=\beta_{1 j}-\mathfrak{x}+k+1, \delta_{j k}=\beta_{2 j}-\mathfrak{x}+k+1, A_{j k}, B_{j k}, C_{j k}, D_{j k}$ are certain constants. To obtain the system of integral equations (6), the Mellin transform

$$
\hat{F}(s)=M[F(x)]=\int_{0}^{+\infty} x^{s-1} F(x) d x
$$

can be applied.

First, let us consider the Mellin transform for the first equation of system (6).

$$
\begin{aligned}
M[ & \left.g_{1 j}^{(0)}\left(\xi_{1}\right)-F_{1 j}^{(0)}\left(\xi_{1}\right)\right] \\
= & M\left[\sum _ { k = 0 } ^ { n - 1 } b _ { 1 j k } ( \xi _ { 1 } ) \left[c_{0}^{(0)}\left(\xi_{1}\right)+\int_{0}^{+\infty} K_{1 j k}^{(22)}(\xi) d_{k}^{(1)}\left(\xi_{2}\right) d \xi_{2}\right.\right. \\
& \left.\left.+\int_{0}^{+\infty} K_{1 j k}^{(11)}(\xi) d_{k}^{(0)}\left(\xi_{2}\right) d \xi_{2}\right]\right] \\
= & \sum_{k=0}^{n-1} M\left[b _ { 1 j k } ( \xi _ { 1 } ) \left[c_{0}^{(0)}\left(\xi_{1}\right)+\int_{0}^{+\infty} K_{1 j k}^{(22)}(\xi) d_{k}^{(1)}\left(\xi_{2}\right) d \xi_{2}\right.\right. \\
& \left.\left.+\int_{0}^{+\infty} K_{1 j k}^{(11)}(\xi) d_{k}^{(0)}\left(\xi_{2}\right) d \xi_{2}\right]\right] .
\end{aligned}
$$

For our convenience, we will consider the Mellin transform of every summand in (7) separately.

$$
M\left[b_{1 j k}\left(\xi_{1}\right) c_{0}^{(0)}\left(\xi_{1}\right)\right]=\int_{0}^{+\infty} \xi_{1}^{s-1} A_{j k} \xi_{1}^{\gamma_{j k}} c_{k}^{(0)}\left(\xi_{1}\right) d \xi_{1}=A_{j k} \hat{c}_{k}^{(0)}\left(s+\gamma_{j k}\right)
$$


For the second and third summands, we introduce the change of variables $\frac{\xi_{1}}{\xi_{2}}=t$, so that $\xi_{1}=t \xi_{2}, d \xi_{1}=\xi_{2} d t$

$$
\begin{aligned}
M & {\left[b_{1 j k}\left(\xi_{1}\right) \int_{0}^{+\infty} K_{1 j k}^{(22)}(\xi) d_{k}^{(1)}\left(\xi_{2}\right) d \xi_{2}\right] } \\
& =\int_{0}^{+\infty} \xi_{1}^{s-1} A_{j k} \xi_{1}^{\gamma_{j k}}\left[\int_{0}^{+\infty} K_{1 j k}^{(22)}(\xi) d_{k}^{(1)}\left(\xi_{2}\right) d \xi_{2}\right] d \xi_{1} \\
& =\int_{0}^{+\infty} \xi_{1}^{s-1} A_{j k} \xi_{1}^{\gamma_{j k}}\left[\int_{0}^{+\infty} K_{1 j k}^{(22)}\left(\frac{\xi_{1}}{\xi_{2}} \xi_{2}, \xi_{2}\right) d_{k}^{(1)}\left(\xi_{2}\right) d \xi_{1}\right] d \xi_{2} \\
& =A_{j k} \int_{0}^{+\infty} \int_{0}^{+\infty} \xi_{2}^{s-1} t^{s-1} \xi_{2}^{\gamma_{j k}} t^{\gamma_{j k}} K_{1 j k}^{(22)}(t, 1) \xi_{2}^{-1} d_{k}^{(1)}\left(\xi_{2}\right) \xi_{2} d \xi_{2} d t \\
& =A_{j k} \int_{0}^{+\infty} t^{s-1} K_{1 j k}^{(22)}(t, 1) t^{\gamma_{j k}} d t \int_{0}^{+\infty} \xi_{2}^{s-1} d_{k}^{(1)}\left(\xi_{2}\right) \xi_{2}^{\gamma_{j k}} d \xi_{2} \\
& =A_{j k} \hat{K}_{1 j k}^{(11)}\left(s+\gamma_{j k}\right) \hat{d}_{k}^{(1)}\left(s+\gamma_{j k}\right) .
\end{aligned}
$$

Analogously, for the third summand.

$$
\begin{aligned}
& M\left[b_{1 j k}\left(\xi_{1}\right) \int_{0}^{+\infty} K_{1 j k}^{(11)}(\xi) d_{k}^{(0)}\left(\xi_{2}\right) d \xi_{2}\right] \\
& \quad=A_{j k} \int_{0}^{+\infty} t^{s-1} K_{1 j k}^{(11)}(t, 1) t^{\gamma_{j k}} d t \int_{0}^{+\infty} \xi_{2}^{s-1} d_{k}^{(0)}\left(\xi_{2}\right) \xi_{2}^{\gamma_{j k}} d \xi_{2} \\
& =A_{j k} \hat{K}_{1 j k}^{(11)}\left(s+\gamma_{j k}\right) \hat{d}_{k}^{(0)}\left(s+\gamma_{j k}\right) .
\end{aligned}
$$

As a result, after the Mellin transform of the first equation (7), we obtain a difference equation of type

$$
\begin{aligned}
\hat{g}_{1 j}^{(0)}(s)= & \hat{F}_{1 j}^{(0)}(s)+\sum_{k=0}^{n-1} A_{j k}\left[\hat{c}_{k}^{(0)}\left(s+\gamma_{j k}\right)\right. \\
& \left.+\hat{K}_{1 j k}^{(22)}\left(s+\gamma_{j k}\right) \hat{d}_{k}^{(1)}\left(s+\gamma_{j k}\right)+\hat{K}_{1 j k}^{(11)}\left(s+\gamma_{j k}\right) \hat{d}_{k}^{(0)}\left(s+\gamma_{j k}\right)\right] .
\end{aligned}
$$

Analogously, we obtain left difference equations from system (6). Finally, our system of integral equations (6) is transformed to the system of difference equations of the form:

$$
\left\{\begin{aligned}
\hat{g}_{1 j}^{(0)}(s)= & \hat{F}_{1 j}^{(0)}(s)+\sum_{k=0}^{n-1} A_{j k}\left[\hat{c}_{k}^{(0)}\left(s+\gamma_{j k}\right)\right. \\
& \left.+\hat{K}_{1 j k}^{(2)}\left(s+\gamma_{j k}\right) \hat{d}_{k}^{(1)}\left(s+\gamma_{j k}\right)+\hat{K}_{1 j k}^{(11)}\left(s+\gamma_{j k}\right) \hat{d}_{k}^{(0)}\left(s+\gamma_{j k}\right)\right] \\
\hat{g}_{1 j}^{(1)}(s)= & \hat{F}_{1 j}^{(1)}(s)+\sum_{k=0}^{n-1}(-1)^{\gamma_{j k}} B_{j k}\left[\hat{c}_{k}^{(1)}\left(s+\gamma_{j k}\right)\right. \\
& \left.+\hat{K}_{1 j k}^{(21)}\left(s+\gamma_{j k}\right) \hat{d}_{k}^{(1)}\left(s+\gamma_{j k}\right)+\hat{K}_{1 j k}^{(12)}\left(s+\gamma_{j k}\right) \hat{d}_{k}^{(0)}\left(s+\gamma_{j k}\right)\right] \\
\hat{g}_{2 j}^{(0)}(s)= & \hat{F}_{2 j}^{(0)}(s)+\sum_{k=0}^{n-1} C_{j k}\left[\hat{d}_{k}^{(0)}\left(s+\gamma_{j k}\right)\right. \\
& \left.+\hat{K}_{2 j k}^{(12)}\left(s+\gamma_{j k}\right) \hat{c}_{k}^{(1)}\left(s+\gamma_{j k}\right)+\hat{K}_{2 j k}^{(1)}\left(s+\gamma_{j k}\right) \hat{c}_{k}^{(0)}\left(s+\gamma_{j k}\right)\right] \\
\hat{g}_{2 j}^{(1)}(s)= & \hat{F}_{2 j}^{(1)}(s)+\sum_{k=0}^{n-1}(-1)^{\delta_{j k}} D_{j k}\left[\hat{d}_{k}^{(1)}\left(s+\gamma_{j k}\right)\right. \\
& \left.+\hat{K}_{2 j k}^{(21)}\left(s+\gamma_{j k}\right) \hat{c}_{k}^{(1)}\left(s+\gamma_{j k}\right)+\hat{K}_{2 j k}^{(22)}\left(s+\gamma_{j k}\right) \hat{c}_{k}^{(0)}\left(s+\gamma_{j k}\right)\right] .
\end{aligned}\right.
$$

Theorem 2 Boundary value problem (1), (3) is uniquely solvable if and only if the system of difference equations (8) is uniquely solvable. 


\section{Conclusion}

The simple difference system of first order was found and described by the author recently [7]. This example shows that even for the simplest cases, we obtain the system of difference equations of first order with variable coefficients. There are no methods for solving such systems, and we need to develop new approaches for this purpose.

\section{Competing interests}

The author declares that he has no competing interests.

\section{Acknowledgements}

The author is very grateful to referees for their remarks and useful suggestions.

Received: 1 March 2013 Accepted: 11 September 2013 Published: 07 Nov 2013

\section{References}

1. Eskin, G: Boundary Value Problems for Elliptic Pseudo Differential Equations. Am. Math. Soc., Providence (1981)

2. Vasil'ev, VB: Wave Factorization of Elliptic Symbols: Theory and Applications. Introduction to the Theory of Boundary Value Problems in Non-Smooth Domains. Kluwer Academic, Dordrecht (2000)

3. Nazarov, SA, Plamenevskij, BA: Elliptic Problems in Domains with Piecewise Smooth Boundaries. de Gruyter, Berlin (1994)

4. Kozlov, VA, Maz'ja, VG, Rossmann, J: Elliptic Boundary Value Problems with Point Singularities. Am. Math. Soc., Providence (1997)

5. Schulze, B-W: Boundary Value Problems and Singular Pseudo-Differential Operators. Wiley, Chichester (1998)

6. Titchmarsh, E: Introduction to the Theory of Fourier Integrals. Chelsey Publishing, New York (1986)

7. Vasilyev, VB: On some difference equations of first order. Tatra Mt. Math. Publ. 54, 165-181 (2013)

10.1186/1687-1847-2013-289

Cite this article as: Vasilyev: General boundary value problems for pseudo-differential equations and related difference equations. Advances in Difference Equations 2013, 2013:289

\section{Submit your manuscript to a SpringerOpen ${ }^{\circ}$ journal and benefit from:}

- Convenient online submission

- Rigorous peer review

Immediate publication on acceptance

Open access: articles freely available online

- High visibility within the field

- Retaining the copyright to your article 